\title{
Funktionen und Dimensionen der Strafrechtsvergleichung
}

\author{
Michael Kubiciel"
}

Rezension zu Beck, Susanne/Burchard, Christoph/Fateh-Moghadam, Bijan (Hrsg.): Strafrechtsvergleichung als Problem und Lösung, 2011, Baden-Baden (Nomos Verlag), ISBN 978-3-8329-5483-3, brosch., 79 Euro
A. Einführung
B. Grundlagendiskurse
I. Methodik und Rechtstheorie....... 213
II. Rechtsvergleich vs. Kulturver- gleich?
C. Zusammenhang zwischen Funktion und Dimension der Rechtsverglei- chung.

D. Aufgaben wissenschaftlicher Rechtsvergleichung ....................... 217

I. Legislative Rechtsvergleichung ..... 217

II. Judikative Rechtsvergleichung ...... 220

E. Fazit ............................... 222

\section{A. Einführung}

Ihre erste Blütephase verdankt die moderne Rechtsvergleichung einer Geisteshaltung, die staatliche und disziplinäre Grenzen überwand. Die mit den Reformen ihrer nationalen Rechtsordnungen betrauten Juristen des 19. Jahrhunderts sahen sich an einem gemeinsamen Werk, dessen Gelingen nach ihrer Überzeugung einen grenzüberschreitenden Austausch von Ideen verlangte. ${ }^{1}$ Heute, mehr als eineinhalb Jahrhunderte später wird der Strafrechtsvergleichung ein neuerlicher Bedeutungszuwachs prophezeit. Dessen Grundlage soll die Internationalisierung und Europäisierung des Strafrechts bilden. Tatsächlich zeigen die Entwicklungen des Völkerstrafrechts, der transnationalen Strafverfolgung und der globalen und regionalen Rechtsharmonisierung einen klaren Trend: Das Strafrecht - einst die domaine réservée der Nationalstaaten - entwickelt sich zu einem trans- und supranationalen Rechtsinstrument. ${ }^{2}$ Diese Entwicklung wird nicht nur die kriminalpolitischen und rechtswissenschaftlichen Diskurse internationalisieren. Sie soll auch der Strafrechtsvergleichung einen erheblichen Bedeutungszuwachs verleihen. ${ }^{3}$ Vor diesem Hintergrund will der zu besprechende Band die Weite der Arbeitsfelder und die Leistungsfähigkeit der Rechtsvergleichung ausmessen, indem „grundlagenorientierte, strafrechtsdogmati-

* Dr. Michael Kubiciel, Wiss. Ass. und Habilitand am Lehrstuhl für Strafrecht, Strafprozessrecht und Rechtsphilosophie an der Universität Regensburg.

1 H.-H. Jescheck, Entwicklung, Aufgaben und Methoden der Strafrechtsvergleichung, Tübingen 1955, S. 10 ff. Am Beispiel L. H. Riemer, Das Netzwerk der ,Gefängnisfreunde' (1830-1872), Frankfurt a.M. 2005, S. 3 ff.

$2 \mathrm{Zu}$ dieser Entwicklung U. Sieber, Strafrechtsvergleichung im Wandel, in: ders./H. J. Albrecht (Hrsg.), Strafrecht und Kriminologie unter einem Dach, Berlin, 2006, S. 78 (80 ff., inbes. S. 90 f.). Zu weiteren Binnendifferenzierungen M. Kubiciel, Book Review: Ordering Pluralism, Criminal Law Forum 22 (2011), 433 (434).

3 So Sieber, Strafrechtsvergleichung (Fn. 2), S. 93. Ebenso S. Beck/C. Burchard/B. Fateh-Moghadam, in: dies., S. 5; Hilgendorf, in: S. Beck/C. Burchard/B. Fateh-Moghadam, S. 11 (12). 
sche und internationalstrafrechtliche Perspektiven" der Strafrechtsvergleichung zusammengeführt werden. ${ }^{4}$

\section{B. Grundlagendiskurse}

\section{Methodik und Rechtstheorie}

Zweifel daran, dass die Rechtsvergleichung auf den prognostizierten Bedeutungszuwachs vorbereitet ist, weckt jene Theorie, die seit Jahrzehnten im Zentrum jeder wissenschaftlichen Debatte um die Rechtsvergleichung steht: die funktionale Methode. ${ }^{5}$ Dass sie eine derartige Bedeutung erlangen konnte, liegt nicht zuletzt an der Mehrdeutigkeit des Begriffs Funktionalismus, der Platz für die unterschiedlichsten, teils einander widersprechenden Konzeptionen lässt. ${ }^{6}$ Angesichts dessen liegt es nahe, zunächst klarzustellen, was die funktionale Methode nicht ist - eine Rechtstheorie. Gleichwohl wird dies nicht selten unterstellt und sodann als Kritik gegen die funktionale Methode gewendet. ${ }^{7}$ Sie erfasse, so heißt es, lediglich Normen und deren Wirkungen und vernachlässige über das geschriebene Recht die Bedeutung der Rechtskultur. ${ }^{8}$ Erst die in den letzten Jahren vollzogene "neoromantische Wende“ habe, meint James Whitman, die Rechtsvergleichung für den Einfluss der Rechtskultur auf die Ausgestaltung des Rechts sensibilisiert ${ }^{9}$ und die Perspektive der Komparatistik verbreitert. ${ }^{10}$

Diese Gegenüberstellung zwischen einem an Normen haftenden Funktionalismus und einer die kulturelle Dimension des Rechts reflektierenden Komparatistik ist so eindrücklich wie überzeichnet. Denn der Funktionalismus steht in der Rechtsvergleichung nur für ein methodisches Vorgehen, das an einem Sachproblem ansetzt und nach funktional äquivalenten Lösungen in den Vergleichsrechtsordnungen Aus-

4 S. Beck/C. Burchard/B. Fateh-Moghadam, in: dies. (Hrsg.), S. 5. - Zudem enthält der Band zwei Beispiele binationaler Rechtsvergleichung: L. Wörner, a.a.O., S. 135 ff.; K. Bruckmüller, a.a.O., S. 179 ff.

5 Vgl. R. Michaels, The Functional Method of Comparative Law, in: M. Reimann/R. Zimmermann (Hrsg.), Oxford Handbook of Comparative Law, Oxford 2006, S. 339 (340).

$6 \mathrm{Zu}$ den unterschiedlichen Konzeptionen Michaels, Functional Method (Fn. 5), S. 343 ff.

7 S. die Betonung der methodischen und rechtstheoretischen Seite des funktionalen Ansatzes durch Graziadie, The functionalist heritage, in: P. Legrand/R. Munday (Hrsg.), Comparative Legal Studies, Cambridge 2003, S. 100.

8 Vgl. etwa D. Richers, Postmoderne Theorie der Rechtsvergleichung?, ZaöRV 67 (2007), S. 509ff.; M. Graziadie, Heritage (Fn. 8), S. 100.

9 So J. Whitman, The neo-Romantic turn, in: Legrand/Munday (Fn. 7), S. 312 f. Zum Einzug rechtskulturellen Denkens in die Rechtsvergleichung R. Cotterrell, Comparative Law and Legal Culture, in: Reimann/Zimmermann, Handbook (Fn. 5), S. 709 (710 ff.).

10 S. dazu Graziadei (Fn. 7), S. 110. 
schau hält. ${ }^{11}$ Diese Arbeitsweise impliziert keine Festlegung auf eine Rechtstheorie, die dem Vergleich zugrunde zu legen wäre. Daher müssen Funktionalisten das Recht nicht mit dem Gesetz identifizieren ${ }^{12}$ oder in Abrede stellen, dass das Recht eine Kulturerscheinung ist. ${ }^{13}$ Vielmehr lässt sich die funktionale Rechtsvergleichung - wie Bijan Fateh-Moghadam zeigt - auch mit einer soziologischen Rechtstheorie vereinbaren, welche die Bedeutung moralischer, ökonomischer, sozialer und kultureller Einflüsse auf das Recht betont (S. 50 f.).

Die Diskrepanzen zeigen, dass die funktionalen Methode keine Rechtstheorie bedingt, sondern eine solche voraussetzt. Die funktionale Komparatistik beruht daher auch nicht auf einer Entscheidung zugunsten einer Rechtstheorie, die das Recht ausschließlich als ein Instrument der sozialen Organisation und Steuerung wahrnimmt (s. aber Bijan Fateh-Moghadam, S. 44). Die bei Zweigert und Kötz ${ }^{14}$ spürbare Präferenz für ein instrumentelles Rechtsverständnis dürfte hauptsächlich dem rechtstheoretischen Großklima der späten 1960er Jahre geschuldet sein, in dem Zweigert und Kötz ihre Arbeit entwarfen. Konzeptionell zwingend ist der Zusammenhang zwischen einer funktionellen Methode und einem funktionalistischen Rechtsverständnis jedoch nicht.

\section{Rechtsvergleich vs. Kulturvergleich?}

Die funktionale Methode und der „Legal-Culture“-Ansatz schließen sich mithin nicht aus, sondern lassen sich miteinander verbinden: Die funktionale Methode ist ein geeignetes Verfahren, um funktional äquivalente und inäquivalente Regeln zu identifizieren; doch sind die Vergleichsgegenstände - wie auch Weigend betont (S. 131) - in vielen Fällen kulturell imprägniert. ${ }^{15}$

Gleichwohl spiegelt sich die Frontstellung zwischen einer funktional orientierten und einer kulturelle Divergenzen berücksichtigenden Rechtsvergleichung auch in den unterschiedlichen Akzentuierungen der Beiträge des Sammelbands wider, die der Theorie der Strafrechtsvergleichung gewidmet sind. So plädiert Eric Hilgendorf (S. 23) dafür, die Perspektive des Rechtsvergleichs auszuweiten und „das Recht konsequent als Teil der Kultur zu verstehen." Auch Susanne Beck meint, die Rechtsvergleichung

11 S. M. Rheinstein, Einführung in die Rechtsvergleichung, 2. Aufl. 1987, S. 15; K. Zweigert/H. Kötz, Einführung in die Rechtsvergleichung, 3. Aufl., München 1996, S. 33 ff. S. ferner A. Eser, in: H. J. Albrecht et. alt. (Hrsg.), Funktionen, Methoden und Grenzen der Strafrechtsvergleichung, Internationale Perspektiven in Kriminologie und Strafrecht - Festschrift für Günther Kaiser, Berlin 1998, S. 1499 (1521); U. Nelles, in: J. Arnold et. alt. (Hrsg.), Rechtsvergleichung im Internet?, Menschengerechtes Strafrecht - Festschrift für Albin Eser, München 2005, S. 1005 (1010).

12 Gegen diese Verkürzung Zweigert/Kötz, Rechtsvergleichung (Fn. 11), S. 25.

13 I. Erg. wie hier Michaels, Functional Method (Fn. 5), S. 362, 381.

14 Diese Dimension des Rechts betont Rheinstein, Rechtsvergleichung (Fn. 11), S. 25 f. Ähnlich Zweigert/ Kötz, Rechtsvergleichung (Fn. 11), S. 45.

15 Dazu P. Legrand, in: D. Nelken/J. Feest (Hrsg.), Adapting Legal Cultures, Oxford 2001, S. 55 (59, 63); S. H. Jung, Recht und kulturelle Identität, ZStW 121 (2009), S. 59 ff. 
müsse sich „von der Konzentration auf das geschriebene Recht“ lösen und das Recht „als Teil der kulturellen Kommunikation" wahrnehmen (S. 79). Daher habe sie die „Kultur in ihrer Gesamtheit“ (S. 71) und damit „Sprache, Geschichte, Wirtschaft, Sozialstrukturen, Moralentwicklungen etc. " in ihr Vergleichsprogramm einzubeziehen (S. 86). Dieser „maximalen Ausweitung des Untersuchungsgegenstands“ begegnet Bijan Fateh-Moghadam mit Skepsis. Ein umfassender Kulturvergleich setze eine Totalperspektive auf die Gesellschaft voraus, für die es in funktional differenzierten Gesellschaften keinen Standort gebe (S. 49 f.). An der funktionalen Methode kritisiert Fateh-Moghadam hingegen die Vernachlässigung der "rechtsinterne Funktion“ und dogmatischen Struktur eines Rechtsinstituts, die Folge der Fokussierung auf die Wirkungsweise eines Rechtsinstitutes sei (S. 47). Diese Kritik ist berechtigt. Wenn der Komparatist lediglich prüft, ob bzw. inwieweit ein tatsächliches Problem rechtlich gelöst wird, erkennt er zivil-, verwaltungs- und strafrechtliche Lösungen implizit als gleichartig und austauschbar an. ${ }^{16}$ Damit geht sein Blick von vornherein an rechtstheoretischen und dogmatischen Feinstrukturen vorbei. Dem Anspruch jeder Komparatistik, Übereinstimmungen und Divergenzen offen zu legen, kann er so nicht gerecht werden.

Es ist daher zu begrüßen, dass Fateh-Moghadam für eine stärkere Berücksichtigung der juristisch-dogmatischen Begründungszusammenhänge eines Rechtsinstituts plädiert (S. 52 f.). Doch steht an deren Ende in vielen Fällen die Rechtskultur. So ist die Ausleuchtung des rechtskulturellen Regelungsumfeldes zur Beurteilung der Umsetzungschancen von supranationalen Harmonisierungsprojekten unerlässlich (Perron, S. 124). Auch bei der rechtsvergleichenden Vorbereitung nationaler Gesetzgebungsverfahren sollte nicht nur auf die Aussonderung von Regelungen „aus Ländern mit einer sehr abweichenden Kultur" geachtet werden (Perron, S. 123). Vielmehr können auch Unterschiede in der außerrechtlichen Feinstruktur für die Gesetzgebung relevant sein: So ist die Ausgestaltung des Wirtschaftsstrafrechts abhängig von der ökonomischen Organisation des Wirtschaftslebens, und die Antworten auf medizinstrafrechtliche Fragen werden von der Sozialethik mitgeprägt. Daher kann man einen anlassbezogenen Partialvergleich des soziokulturellen Regelungsumfeldes einer Norm für erforderlich halten, selbst wenn man mit Fateh-Moghadam der Meinung ist, dass ein kultureller Totalvergleich weder durchführbar noch notwendig ist.

\section{Zusammenhang zwischen Funktion und Dimension der Rechtsvergleichung}

$\mathrm{Zu}$ entscheiden ist damit, in welchen Fällen den Regelungszusammenhängen einer Norm bis in das juristische Vorfeld der Kultur nachzuspüren ist. Insbesondere die neoromantischen Gegner der funktionalen Komparatistik erwecken den Eindruck, der Rechtsvergleicher stehe stets vor der binären Entscheidung zwischen einer geset-

16 So Zweigert/Kötz, Rechtsvergleichung (Fn.11), S.45; ähnlich Rheinstein, Rechtsvergleichung (Fn. 11), S. 15. 
zesfokussierten und einer kulturorientierten Sichtweise und müsse sich in jedem Fall für die letztgenannte Perspektive entscheiden. Dieser Eindruck trügt. ${ }^{17}$ Denn zum einen bilden nicht alle Rechtsnormen Kulturnormen ab: Eine moderne Gesellschaft mit ihren vielfältigen Steuerungsproblemen ist, wie schon M.E. Mayer wusste, auf Rechtsnormen angewiesen, denen ein unmittelbarer Bezug zur Rechtskultur fehlt. ${ }^{18}$ Zum zweiten - und dies ist entscheidend - hängt die Dimension von der Funktion der Rechtsvergleichung ab, wie sich thesenhaft zugespitzt formulieren ließe. Die Aufgabe des Rechtsvergleichs gibt also nicht nur die Methode, ${ }^{19}$ sondern auch die Tiefe des Vergleichs vor. ${ }^{20}$ Daher kann der Komparatist nicht mit einer fixen Optik arbeiten, sondern hat jene Tiefenschärfe zu wählen, die dem Zweck des konkreten Vergleichs angemessen ist. Die rechtsvergleichende Feststellung einer Tatortstrafbarkeit i.S.d. $\$ 7$ StGB erfordert beispielsweise nur eine Analyse des geltenden Rechts. Hingegen muss der Blick des Rechtsvergleichers über das Recht hinaus auf die Kultur einer Gesellschaft gelenkt werden, wenn er politikberatend tätig wird. Hier ist die Feststellung der funktionalen Äquivalenz nur der eine Teil der Aufgabe. Der andere Teil besteht in der Erörterung der Frage, ob die unterschiedlichen Regelungsmodelle derart mit ihrer Rechtskultur verflochten sind, dass ihr Import Akzeptanzprobleme bereiten könnte. Weist eine Norm einen solchen Bezug auf, muss der Rechtsvergleich in einem partiellen Kulturvergleich münden, wenn „legal transplants" nicht von der heimischen Umgebung abgestoßen werden sollen.

Die Beispiele deuten an, dass die Rechtsvergleichung - anders als die funktionale Methode unterstellt - nicht allein der Identifizierung von funktional äquivalenten Rechtsregeln dient. Die Rechtsvergleichung hat vielmehr unterschiedliche Aufgaben, von denen abhängt, ob der Vergleich nur die Funktionsweise einer Norm, deren dogmatische Struktur oder auch "die“ Kultur erfassen muss. So gesehen erscheint nicht nur die Gegenüberstellung von funktionaler Methode und Rechtskultur überzeichnet. Es wird auch deutlich, dass beide Positionen nur Einzelausschnitte der Rechtsvergleichung erfassen. Folglich kann kein Ansatz für sich in Anspruch nehmen, eine Theorie der Rechtskomparatistik zu sein. ${ }^{21}$

17 Eine schroffe Gegenüberstellung ablehnend Perron, in: S. Beck/C. Burchard/B. Fateh-Moghadam, S. 121.

18 S. schon M.E. Mayer, Rechtsnormen und Kulturnormen, Berlin 1903, S. $19 \mathrm{ff}$.

19 Dazu Eser, Funktionen (Fn. 11), 1520; Jescheck, Entwicklung (Fn. 1), S. 36; Nelles, Rechtsvergleichung (Fn. 11), S. 1010.

20 Zum Zusammenhang von Aufgabe und Reichweite des Vergleichs Sieber, Strafrechtsvergleichung (Fn. 2), S. $128 \mathrm{f}$.

21 Dieser Befund bestätigt die Forderungen nach der Entwicklung einer Theorie der Rechtsvergleichung; s. etwa die Forderungen von Hilgendorf (Fn. 3), S. 24; Sieber, Strafrechtsvergleichung (Fn. 2). S. 126 ff. 


\section{Aufgaben wissenschaftlicher Rechtsvergleichung}

Jeder wissenschaftliche Rechtsvergleich dient einem Zweck. Erst der Zweck gibt der Suche eine Richtung und den gewonnenen Daten Sinn. ${ }^{22}$ Dass die Rechtsvergleichung praktischen Zwecken dient, ist daher nicht kritikwürdig. ${ }^{23}$ Die Rechtswissenschaft darf sich zur Erfüllung ihrer dogmatischen und rechtspolitischen Aufgaben der Rechtsvergleichung als einer Methode bedienen. ${ }^{24}$ Erst dann, wenn die Rechtswissenschaft jene methodischen Standards verletzt, die sie als Wissenschaft zu erfüllen hat, sinkt auch die Rechtsvergleichung auf den Status einer reinen Dienstleistung ab.

Besonders groß ist die Gefahr, wissenschaftliche Standards zu unterlaufen, auf dem Feld der Rechtspolitik. ${ }^{25}$ Daher muss sich gerade die legislative Rechtsvergleichung der Grenzen ihrer Möglichkeiten bewusst sein, soll nicht Politik unter dem Deckmantel der Wissenschaft betrieben werden (I.). Aber auch die justizielle Rechtsvergleichung steht vor erheblichen methodischen Herausforderungen (II.).

\section{Legislative Rechtsvergleichung}

Die beiden dem europäischen Strafrecht gewidmeten Beiträge gießen eine gute Portion Wasser in den Wein derer, die mit der Europäisierung des Rechts auch die Stunde der Rechtsvergleichung gekommen sehen. Während Frank Meyer zeigt, dass die Strafrechtsvergleichung wichtige europäische Regulierungsfragen nicht beantworten kann, verweist Pierre Hauck darauf, dass der europäische Gesetzgeber die Rechtsvergleichung nicht zu Rate ziehen will.

a) Frank Meyer widmet seinen Beitrag dem europäisierten Strafprozessrecht. Er legt dar, dass europäische Rechtshilferegeln die Mitgliedsstaaten der EU zunehmend in die Lage versetzen, Hoheitsgewalt außerhalb ihres Territoriums auszuüben (S. 94 ff.). Mit dieser Erweiterung der staatlichen Befugnisse, so Meyer mit Recht (S. 98), habe die Entwicklung der Verfahrensrechte der Betroffenen nicht Schritt gehalten. ${ }^{26}$ Die Frage, wie diese Defizite zu beheben seien, könne die Rechtsverglei-

22 Alles andere führte zu dem von Jescheck, Entwicklung (Fn. 1), S. 25, beschriebenen „ziellosen Schwimmen" in dem Meer des Materials.

23 S. aber Monas Kritik an dem „Servicemodell“ (Mona, in: S. Beck/C. Burchard/B. Fateh-Moghadam, S. 103 ff.), der freilich an späterer Stelle (S. 109) klarstellt, dass die Rechtsvergleichung „,nicht völlig losgelöst von überhaupt möglichen Zwecken und Zielen" betrieben werden dürfe.

24 Zur Rechtsvergleichung als Methode Jescheck, Entwicklung (Fn. 1), S. 36 f. - Zu den Aufgaben der Rechtsvergleichung Eser (Fn. 11), 1506 ff.; H. Jung, Grundfragen der Strafrechtsvergleichung, JuS 1998, S. 1 ff.; Nelles, Rechtsvergleichung (Fn. 11), S. 1009 f.; Sieber, Strafrechtsvergleichung (Fn. 2), S. $94 \mathrm{ff}$.

25 Den Unterschied zwischen Rechtsvergleichung und Rechtspolitik betonen Rheinstein, Rechtsvergleichung (Fn. 12), S. 26; Sieber, Strafrechtsvergleichung (Fn.2), S. 120. - S. auch U. Kindhäuser, Die deutsche Strafrechtsdogmatik zwischen Anpassung und Selbstbehauptung, ZStW 121 (2009), S. $954 \mathrm{ff}$.

26 Ausf. dazu B. Noltenius, Strafverfahrensrecht als Seismograph der Europäischen Integration, ZStW 122 (2010), S. $604 \mathrm{ff}$. 
chung indes nicht beantworten, denn der traditionelle Verfahrensschutz in Nationalstaaten lasse sich nicht auf das in der EU entstehende Mehrebenensystem übertragen (S. 100). Freilich basiert der Befund, dass die Rechtsvergleichung für diese Zukunftsfragen keine Antworten bereit halte (S. 102), auf einem Vergleich von strafprozessualen Eingriffs- und Schutzsystemen in Nationalstaaten mit dem institutionellen und rechtlichen Gefüge von Mehrebenensystem. Dies zeigt, dass die Vergleichsmethode zumindest ein geeignetes Diagnosemittel ist. Denkbar ist es zudem, dass die in der Praxis der transnationalen Strafverfolgung eingesetzte Rechtsvergleichung verfahrensrechtliche Mindeststandards herausfiltert, wie Christoph Burchard in seinem äußerst lesenswerten Beitrag zur Europäischen Ermittlungsanordnung zeigt (S. 293 f.). ${ }^{27}$ Doch dürfte damit lediglich eine kleine Schnittmenge europaweit anerkannter Lösungen zu Tage gefördert werden. In allen anderen Regulierungsfragen kann der Komparatist dem Gesetzgeber ebenso wenig wie jeder andere Vertreter der Rechtswissenschaft sagen, welche Entscheidung er zu treffen hat. Er kann die Entscheidung des Gesetzgebers lediglich vorbereiten. Darin unterscheiden sich nationalstaatliche nicht von europäischen Gesetzgebungsverfahren.

b) Was kann die Rechtsvergleichung bei der Vorbereitung rechtspolitischer Entscheidungen leisten? Zweifellos möglich ist es ihr, die in der Welt verbreiteten Lösungsmodelle mitsamt ihrer Vor- und Nachteile zu benennen, ${ }^{28} \mathrm{dem}$ Gesetzgeber also einen „Lösungsvorrat“ zur Verfügung zu stellen (Vogel, S. 205). Bei Harmonisierungsprojekten, namentlich jenen der EU, kommt ihr darüber hinaus die Prüfung zu, ob die Strafbarkeitslücken und Vollzugsdefizite in den Mitgliedsstaaten tatsächlich bestehen. Auf diesen Aspekt der Rechtsvergleichung weist Pierre Hauck mit Recht hin (S. 268). Doch ist die Strafrechtsvergleichung nicht nur wegen der Geltung des gemeinschaftsrechtlichen Subsidiaritätsgrundsatzes in der europäischen Gesetzgebung „programmatisch angelegt“ (S. 268). Vor allem zum Schutz der in der Grundrechte-Charta verbürgten Freiheitsrechte kann die europäisch initiierte Strafrechtssetzung weder Selbstzweck noch Mittel politischer Profilierung sein..$^{29}$ Es ist daher das unionsrechtliche Gebot, die Strafrechtsharmonisierung mit den Freiheitsrechten auszutarieren, das den europäischen Gesetzgeber zur Subsidiaritätsprüfung anhält. Auch hier ist die Rechtsvergleichung ein unerlässliches Diagnosemittel. In der „eurokratisch-tagesgeschäftlichen Rechtssetzung “ bleibt es jedoch viel zu häufig ungenutzt, wie Hauck mit Recht konstatiert (S.271). Stattdessen entwickelt sich eine vom Vogel eindringlich beschriebene (S.207ff.) - Tendenz zur politischen Instrumentalisierung der Rechtsvergleichung: Ziel dieser von der EU gesteuerten Auftrags-

27 Burchard, in: S. Beck/C. Burchard/B. Fateh-Moghadam, S. 275 ff., bezeichnet die im Zuge der Europäischen Ermittlungsanordnung notwendig werdende Rechtsvergleichung treffend als ,exekutorische Rechtsvergleichung".

28 U. Sieber, Rechtliche Ordnung in einer globalen Welt, Rechtstheorie 41 (2010), S. 151 (196).

29 Zum Zusammenhang von Strafrechtsharmonisierung und Freiheitsschutz M. Kubiciel, Das „Lissabon"-Urteil und seine Folgen für das Europäische Strafrecht, GA 2010, 99 (110 ff.). 
forschung sei es, Argumente zu generieren, „um sie als Begründung für (kriminal-)politische Initiativen heranzuziehen“ (S. 207), namentlich: „Harmonisierungsund Handlungsbedarf auf Unionsebene zu begründen“ (S. 212).

c) Will sich die Strafrechtswissenschaft dieser Tendenz entziehen, muss sie sich kritisches Potenzial erarbeiten. Geleitet von dieser Intention empfiehlt Mona der Rechtsvergleichung sich „in ein Verhältnis zur Rechtsphilosophie, mithin zur Frage nach der Gerechtigkeit,“ zu setzen“ (S. 104), mehr noch: die Rechtsvergleichung „in eine umfassende Theorie der Gerechtigkeit" einzugliedern (S.115). Er trägt damit dem Umstand Rechnung, dass die (Straf-)Rechtswissenschaft die Legitimität das geltende Recht nicht prüfen kann, ohne sich auf eine vorpositive Ebene zu begeben. Zwei Gerechtigkeitskonzeptionen erscheinen Mona vielversprechend: die Amartya Sens und jene John Rawls'. Beide Konzeptionen haben neben unbestreitbaren Vorzügen - aber auch Schwächen. So verzichtet Sens Ansatz einer „comparative justice“ zwar auf die Festlegung eines umfassenden, universell gültigen Gerechtigkeitsideals und trägt damit der kulturellen Kontingenz von Gerechtigkeitsüberzeugungen Rechnung. Jedoch muss sich Sen auf die Identifizierung von Handlungsweisen beschränken, die weltweit als „manifeste Ungerechtigkeiten” angesehen werden. ${ }^{30}$ Weniger geeignet ist sein Verfahren daher für die Bewältigung des Gros' kriminalpolitischer Probleme, das außerhalb des schmalen Bereichs flagranten Unrechts angesiedelt ist. Dieses Alltagsgeschäft internationaler und nationaler Gesetzgebung kann Rawls' Methode eines reflexiven Gleichgewichts ${ }^{31}$ zwar erleichtern. Doch setzt Rawls' Verfahren die eigenen Begründungsobjekte - konkrete Gerechtigkeitsprinzipien - voraus. ${ }^{32}$ Folglich muss die Strafrechtswissenschaft jene (Straf-)Rechtsprinzipien und strafrechtliche Legitimationsanforderungen benennen, die in das Verfahren zur Herstellung eines reflexiven Gleichgewichts einzuspeisen sind.

d) Anstatt bei der Bewertung rechtsvergleichend gewonnener Modelle auf abstrakte Gerechtigkeitskonzeptionen zu rekurrieren, ${ }^{33}$ sollte die Strafrechtswissenschaft daher eine Stufe tiefer ansetzen: bei jenen Theorien, die (national und international) zur Legitimation des Strafrechts verwendet werden. Aus ihnen lassen sich die faktischen Anwendungs- und Legitimationsvoraussetzungen der Strafe ableiten, die beispielsweise zeigen, unter welchen Bedingungen das Strafrecht als interkulturelles Werkzeug eingesetzt werden darf. ${ }^{34}$ Nicht nur hier können sich Rechtskomparatistik und

30 A. Sen, The Idea of Justice, London 2009, S. 21, 26.

31 J. Rawls, Eine Theorie der Gerechtigkeit, Frankfurt a.M. 1979, S. 37 f. S. dazu J. Braun, Rechtsphilosophie im 20. Jahrhundert, München 2001, S. $133 \mathrm{f}$.

32 M. Mablmann, Rechtsphilosophie und Rechtstheorie, 2. Aufl., Baden-Baden 2012, $\mathbb{1} 14$ Rn. 41.

33 Zweifel an dem Rekurs auf ideale Gerechtigkeitskonzeptionen hegt auch Weigend, in: S. Beck/C. Burchard/B. Fateh-Moghadam, S. 131 (132). S. ferner J. Vogel, Die Strafgesetzgebungskompetenzen der Europäischen Union nach den Art. 83, 86, 325 AEUV, in: K. Ambos (Hrsg.), Europäisches Strafrecht post-Lissabon, Göttingen 2011, S. 49 (55).

34 Dazu Kubiciel, Strafrechtswissenschaft und europäische Kriminalpolitik, ZIS 2011, S. 742. 
Strafrechtstheorien ergänzen. Auch die Analyse und Evaluierung der von Sieber ${ }^{35}$ eindringlich beschriebenen Veränderungen des Strafrechts durch eine primär präventionsorientierte internationale und europäische Strafrechtssetzung kommt nicht ohne den Rückgriff auf die Straftheorien aus. Denn wenn die Rechtswissenschaft diese Entwicklungen beschreiben und bewerten will, muss sie zuallererst die Frage stellen, ob diese Innovationen noch als Fortschreibungen dessen gelten können, was als „Strafrecht“ bezeichnet wird. ${ }^{36}$ Angesichts dessen ist es zu bedauern, dass das Potenzial dieser Grundlagentheorien für die Analyse der internationalen Strafrechtsentwicklung bislang kaum ausgelotet worden ist. Auch der vorliegende Band widmet ihnen keine besondere Aufmerksamkeit.

\section{Judikative Rechtsvergleichung}

a) Nicht nur auf dem Gebiet der wissenschaftlichen Politikberatung, auch für die Rechtsanwendung wird der Rechtsvergleichung Wachstumspotenzial attestiert. ${ }^{37}$ Diese Prognose kontrastiert indes mit Edward Schramms Befund zur Rolle der Rechtsvergleichung in der deutschen höchstrichterlichen Rechtsprechung (S. 155 ff.): Dort scheint die Rechtsvergleichung ein selten verwendetes Instrument zu sein. ${ }^{38}$ Vor allem aber macht die Lektüre der von Schramm zitierten Urteilspassagen deutlich, dass die Rechtsprechung nicht selten tendenziös, d.h. ergebnisgeleitet auf ausländische Rechtsordnungen zurückgreift. Wenn etwa im Minderheitenvotum des Urteils über die Verfassungswidrigkeit der Fristenlösung beim Schwangerschaftsabbruch auf vergleichbare Regelungen in Österreich, Frankreich, Dänemark und Schweden hingewiesen wird, ${ }^{39}$ erhebt sich unwillkürlich die Gegenfrage, weshalb andersartige Regelungen in anderen europäischen Ländern unerwähnt bleiben. Auch der in das Verfahren zur Verfassungskonformität des Verbots des Geschwisterinzests eingeführte Vergleich von Ländern mit und ohne einem entsprechenden Straftatbestand (Ergebnis: dreizehn zu sieben) stützt weder die eine noch die andere Rechtsauffassung, sondern zeichnet eher das Bild einer „divergenten“ Rechtslage, wie es im Minderheitsvotum treffend heißt. ${ }^{40}$

35 U. Sieber, Grenzen des Strafrechts, ZStW 119 (2007), S. 1 (3 ff.).

36 Näher zu der Bedeutung der Straftheorien Kubiciel, Strafrechtswissenschaft (Fn. 34), S. 744 ff. S. ferner M. Böse, Die Entscheidung des Bundesverfassungsgerichts zum Vertrag von Lissabon und ihre Bedeutung für die Europäisierung des Strafrechts, ZIS 2010, S. 76 (92); J. Vogel, Strafrecht und Strafrechtswissenschaft im internationalen und europäischen Rechtsraum, JZ 2012, 25 (31).

37 S. Beck/C. Burchard/B. Fateh-Moghadam, in: dies., S. 5; Schramm, in: S. Beck/C. Burchard/B. FatehMoghadam, S. 155 (177).

38 Über die von Schramm genannten Entscheidungen hinaus hätte man noch auf die Entscheidung BGHSt 1, $293 \mathrm{ff}$. hinweisen können, in welcher das damals geltende Verbot homosexueller Betätigung (auch) mit Hilfe rechtsvergleichender Überlegungen restriktiv ausgelegt wurde.

39 BVerfGE 39, 1, 94.

40 BVerfGE 120, 224, 230, 258. 
b) Die Beispiele verdeutlichen die Gefahr, dass rechtsvergleichende Daten lediglich zur vordergründigen Absicherung eines anderweitig gefundenen Ergebnisses herangezogen werden. Die Verweise auf das ausländische Recht haben dann keinen Begründungs-, sondern lediglich ornamentalen Darstellungswert; sie fungieren als Mittel zur rhetorisch-topischen Absicherung einer Entscheidung. ${ }^{41}$ Die aus einer solchen Argumentationsweise resultierenden Rationalitätsverluste hat bereits Theodor Viehweg deutlich gemacht. Die Topik, so Viehweg, erleichtere zwar die Argumentation, könne aber ohne eine systematische Einkleidung nur zu fragmentarischen Einsichten führen. ${ }^{42}$ Daher solle nicht der rhetorische Nutzen die Verwendung von Topoi leiten, sondern ein „Ableitungssystem“. ${ }^{43}$ Erst dort, wo dessen Aussagekraft endet, dürfe der Rechtsanwender zur Rhetorik greifen. So gesehen kann auch die rechtsvergleichende Methode der Rechtspraxis nicht ohne weiteres empfohlen werden. ${ }^{44} \mathrm{BGH}$ und BVerfG haben das deutsche Recht fortzuschreiben und dürfen daher rechtsvergleichende Daten (wie andere Topoi auch) nur verwenden, solange sie an systematisch-dogmatische Begründungszusammenhänge zurückgebunden werden können.

c) Wo systematische Begründungszusammenhänge (noch) fehlen, ist der Rückgriff auf die Topik unvermeidlich. Was dies für die Rechtsvergleichung bedeutet, wird bei Boris Burghardt deutlich. Anschaulich zeigt er am Beispiel einer Entscheidung des Internationalen Strafgerichtshofs (IStGH), wie selektiv der IStGH rechtsvergleichend gewonnenes Material verwendet, um die Figur der Täterschaft kraft organisatorischer Machtapparate in das IStGH-Statut hineinzulesen (S. 245 ff.). Die Rolle, die der Rechtsvergleichung von der Rechtspraxis damit zugewiesen wird, ist nach Auffassung Burghardts die einer Beschafferin „diskursiver Legitimität“ (S. 251). Die Rechtsvergleichung sorge dafür, dass die von einem Gericht zur Behandlung eines Sachproblems gewählte Lösung in einer Weise kommuniziert wird, die auch für denjenigen verständlich sei, der das internationale Recht nicht kenne oder ablehne. Auf diese Weise erhöhe die Rechtsvergleichung die „Akzeptanz- und Anschlussfähigkeit der völkerstrafrechtlichen Praxis. " Burghardt macht also aus der systematischen Not neuer Rechtssysteme eine topische Tugend und gibt damit der Praxis der Völkerstrafrechtsprechung ein methodologisches Fundament. Indes bleiben Zweifel daran bestehen, dass sich Akzeptanz und Anschlussfähigkeit topisch-rhetorisch generieren lassen: Ein kenianischer Angeklagter muss eine der deutschen und spanischen Dogmatik entlehnte Begründungsfigur nicht verstehen und er darf sie solange begründet

41 Zum dementsprechenden Umgang der Rechtsprechung mit Literaturstimmen Th. Fischer, Strafrechtswissenschaft und strafrechtliche Rechtsprechung - Fremde seltsame Welten, Festschrift für Rainer Hamm, Berlin 2008, S. $63 \mathrm{ff}$.

42 T. Viehweg, Topik und Jurisprudenz, 5. Auflage, München 1974, S. 31 ff. Zur Rezeption T. Walter, Kleine Rhetorikschule für Juristen, München 2009, S. $49 \mathrm{ff}$.

43 Viehweg, Topik (Fn. 42), S. 44.

44 So aber Schramm, in: S. Beck/C. Burchard/B. Fateh-Moghadam, S. 155 (177); krit. dazu Vogel, in: S. Beck/C. Burchard/B. Fateh-Moghadam, S. 205 (206). 
zurückweisen, wie er Rechtsordnungen anzuführen vermag, die eine Täterschaft qua Nutzung von Machtapparaten nicht (aner-)kennen.

d) Wie das Völkerstrafrecht und die Völkerstrafrechtspraxis diesem Problem entkommen können, deutet Lars Büngener an, der sich mit der schwankenden Argumentation internationaler Gerichte in völkerstrafprozessualen Fragen beschäftigt (S. 215 ff.). Ein völkerstrafrechtliches Verfahren könne langfristig nur funktionieren, wenn es Beschuldigte fair behandele. Eine faire Behandlung, betont Büngener zu Recht, setze aber zunächst eine Definition der Ziele des Völkerstrafrechts voraus. In der Tat: Wer einen kenianischen Milizenchef im Namen der Völkergemeinschaft bestrafen will, schuldet ihm eine Erklärung dafür, weshalb er diese Strafe dulden muss. Die Antwort auf diese Grundlagenfrage kann nicht nur als systematischer Bezugspunkt für die Entwicklung der Völkerstrafrechtsdogmatik fungieren; sie kann auch vorstrukturieren, welches rechtsvergleichende Material für die Lösung konkreter Probleme herangezogen werden kann und welches auszusondern ist.

\section{E. Fazit}

Der Band vermittelt einen umfassenden und realistischen Eindruck von den Arbeitsfeldern der heutigen Strafrechtsvergleichung. Es wird zudem deutlich, dass die Theorie der Rechtsvergleichung einer Fortentwicklung bedarf. Wie oben gezeigt, bietet keine der beiden heute diskutierten Großtheorien eine umfassende Erklärung für die Praxis der Rechtsvergleichung. Für die notwendige Entwicklungsarbeit, die insbesondere das DFG-Projekt „Die Rolle der Strafrechtsvergleichung bei der Europäisierung des Strafrechts" leisten soll, stellt das vorliegende Werk eine vorzügliche Grundlage bereit. Fortentwicklungen sind nach hiesiger Einschätzung in zwei Richtungen möglich und aussichtsreich: Zum einen ist der Zusammenhang zwischen den Funktionen und Dimensionen der Rechtsvergleichung stärker auszuleuchten. Zum anderen sollte das Potenzial eines straftheoretischen Grundlagenvergleichs für die Beschreibung und Bewertung internationaler Rechtsentwicklungen ausgelotet werden.

Ob die Internationalisierung des Strafrechts zu einer Blüte oder nur zu einer Scheinblüte der Rechtsvergleichung führt, ist freilich nicht ausgemacht. Dies hängt auch auf von jenen institutionellen Bedingungen ab, auf die die Rechtsvergleichung ebenso angewiesen ist wie jede andere wissenschaftliche Disziplin. Dass die Strafrechtsvergleichung aber gewillt ist, ihre Aufgaben als Wissenschaft und nicht als nützliches Werkzeug der Rechtspolitik wahrzunehmen, davon legt der zu besprechende Band ein eindruckvolles Zeugnis ab. 